\title{
Mean-field approach to diffusion with interaction: Darken equation and numerical validation
}

\author{
M. Di Pietro Martínez and M. Hoyuelos* \\ Instituto de Investigaciones Físicas de Mar del Plata (IFIMAR - CONICET) and \\ Departamento de Fúsica, Facultad de Ciencias Exactas y Naturales, Universidad Nacional de Mar del Plata. \\ Deán Funes 3350, 7600 Mar del Plata, Argentina
}

(Dated: August 6, 2018)

\begin{abstract}
A mean field theory for diffusion with interaction was introduced in Phys. Rev. E 92, 062118 (2015). Interaction effects are represented with a mean field potential. Here we show that the potential can be directly related to the activity coefficient. In this context, we obtain an alternative derivation of the Darken equation, that relates collective diffusion coefficient and single particle diffusion coefficient (generally different in the presence of interactions). We also carry out a validation test of the model using, as a case study, effective interactions that reproduce Bose-Einstein statistics.
\end{abstract}

PACS numbers: 05.40.-a, 05.60.-k, 66.10.Cg, 05.10.Gg

\section{INTRODUCTION}

A diffusion process is characterized by the collective diffusion coefficient $D$ and the single particle, or tracer, diffusion coefficient $D^{*}$. The former is associated to Fick's first law; it is the proportionality factor between current and concentration gradient: $J=-D \nabla c$; collective behavior is represented by this law. The latter indicates how mean square displacement increases with time: MSD $=2 d D^{*} t$, where $d$ is the system's dimension; in this case, observation of the motion of an individual particle is required. In the absence of interactions, both coefficients are equal. The effects of interactions are, however, different for $D$ or $D^{*}$ and, in general, we have that $D \neq D^{*}$.

In 1948, Darken [1] presented his classical article on diffusion on binary systems in which he introduced equations that relate tracer velocity, collective diffusion coefficient and single particle diffusion coefficient. Darken made the assumption that the solid mixture is composed of two atomic species that have the same atomic volume, so that the system's volume does not change during the process. The starting point of the Darken's approach is the linear phenomenological relationship between particle current and the associated thermodynamic force: the chemical potential gradient. Sridhar [2] stresses the generality of Darken's treatment, that has been applied not only to metallic melts but also, for example, to ceramics, polymers, and molecular diffusion in zeolite. Here, we focus our attention on collective and single particle diffusion coefficients, and show that the relationship can be derived for a system of diffusing particles in which no assumption is made about the system's atomic volume or the particle's environment; it is assumed, instead, that the characteristics of the environment do not depend on the concentration of the diffusing particles. This assumption implies that the description can be reduced to the diffusion of only one species in a stationary environment.

\footnotetext{
* hoyuelos@mdp.edu.ar
}

For a system composed of solute and solvent, the chemical potential of the solute (concentration basis) is

$$
\mu=\mu^{\mathrm{ref}}+R T \ln \left(\gamma c / c_{0}\right)
$$

where $c$ is the molar concentration, $c_{0}$ is a reference concentration, $\mu^{\text {ref }}$ is the chemical potential in an appropriate reference state, and $\gamma$ is the activity coefficient; see, e.g., $[3$, ch. 7$]$ or $[4$, ch. 5]. The departure from ideal behavior of the chemical potential, due to interactions, is represented by the activity coefficient. The activity coefficient $\gamma$ appears in the Darken equation that connects the coefficients $D$ and $D^{*}[1,5]$ in the following way:

$$
D=D^{*}\left(1+\frac{\partial \ln \gamma}{\partial \ln c}\right)
$$

There are, actually, two Darken equations [1]; the first one for the marker velocity and the second one for a combination of the diffusivities of both species that, in turn, is derived from Eq. (2).

In a broader context, we want to mention the seminal work of Batchelor [6], where inhomogeneous suspension of different species of spherical particles, that interact repulsively, is analyzed; let us note that in this case, particles are much larger than the solvent molecules. See also [7-18]. As long as the interaction range is much smaller than the characteristic length of the space dependent concentration, a continuous description based on local differential equations can be introduced (see, for example, [19, 20]).

An understanding of the general problem of diffusion with interaction is of fundamental importance in a large spectrum of processes. For example, during recent decades adatom diffusion (and its interactions) has attracted special attention due to its relevance in dynamic processes on surfaces, such as chemical reactions or growth of epitaxial layers [21-26].

In Ref. [27], a mean field theory was proposed for the problem of diffusion with interaction. (The mean field approximation, i.e., the decorrelation of two-particle joint distributions, was also used, for example, in [28-30].) As 
explained in [27], it can be applied to surface diffusion or to a solute-solvent system. Interaction effects are represented by a (concentration dependent) mean field potential $V$. It corresponds to an effective potential for one particle produced by the rest of the system. In Sect. II we show that there is a direct relationship between the potential $V$ and the activity coefficient $\gamma$. Using this relationship, we also show that the Darken equation (2) can be derived in the context of the mean field theory.

In Sect. III we test the mean field theory with interactions that reproduce Bose-Einstein statistics in equilibrium. This is a particularly difficult test for the mean field approach due to the large fluctuations of boson's equilibrium concentration. In particular, we show that the method reproduces correct results although the Ginzburg criterion is far from being fulfilled. We show that this is due to the fact that the Ginzburg criterion was proposed for the validity of a mean field theory for systems with a phase transition, while no phase transition is present in our case.

\section{DARKEN EQUATION FROM MEAN FIELD THEORY}

We consider a system divided in cells of length $a$. The cell length is much smaller than the characteristic length of space variations of the concentration. We also consider that the interaction range is small enough in order to neglect interactions among particles that are in different cells, so that the interaction is local. There is a time scale given by the average time taken by a particle to jump to a neighboring cell; we write it as $1 / P$, where $P$ is the rate of jump attempts. Both $a$ and $P$ are artificial constructs proposed for a description at a microscopic level; the relevant physical quantity is the combination $P a^{2}$, that corresponds to the free diffusion coefficient $D_{0}$.

There is an average number of particles $n$ in each cell, that is related to the molar concentration by $c=$ $n /\left(N_{A} a^{d}\right)$, where $N_{A}$ is Avogadro's constant and $d$ is the system dimension. For simplicity, we consider $d=1$, but the calculations are easily extended to higher dimensions. We consider normal diffusion, i.e., situations where the mean square displacement is proportional to time (this excludes hard-core interaction in 1D).

The first step is to obtain the transition probability $W_{i, i+1}$ for a particle that goes from cell $i$ to a neighboring cell $i+1$, in terms of mean field potentials $V_{i}$ and $V_{i+1}$; $V_{i}$ is an abbreviation of $V\left(n_{i}\right)$, where $n_{i}$ is the number of particles in cell $i$. The main assumptions used for the derivation of $W_{i, i+1}$ are detailed balance and that the transition probabilities take the general form of the Arrhenius formula (see Ref. [27] for more details). The resulting transition probability is

$$
W_{i, i+1}=P e^{-\beta\left[(\theta-1) V_{i}+(\theta+1) V_{i+1}+\Delta U\right] / 2},
$$

where $P$ is the rate of jump attempts; $\beta=1 / k_{B} T$; $\Delta U=U_{i+1}-U_{i}$ is an external potential change due, for example, to a gravitational field; and $\theta$ is an interpolation parameter that takes values between -1 and 1 (a parameter $\gamma$ is used in Ref. [27]; here we use $\theta$ in order to avoid confusion with the activity coefficient). If $\theta=0$, the transition probability depends on the difference $V_{i+1}-V_{i}$; if $\theta=-1$, it depends on the potential in the origin cell $V_{i}$; and if $\theta=1$, it depends on the potential in the target cell $V_{i+1}$.

Parameter $\theta$ (in general a function of the concentration c) appears in the transition probabilities as a consequence of the mentioned assumptions of detailed balance and the general form of the Arrhenius formula, as shown in Ref. [27]. A frequent choice in Monte Carlo simulations of diffusion processes is the Glauber algorithm, in which the transition probabilities depend on the difference of the potential, $\Delta V=V_{i+1}-V_{i}$. This choice corresponds to $\theta=0$; it guarantees evolution to equilibrium but does not guarantee a realistic representation of the transient evolution [31]. An extra parameter is necessary in order to correctly describe the out of equilibrium behavior, characterized by the diffusion and mobility coefficients; this is the role of $\theta$. The transition probability (3) can also be written as

$$
W_{i, i+1}=P \exp \left[-\beta\left(\theta \frac{V_{i+1}+V_{i}}{2}+\frac{\Delta V}{2}+\frac{\Delta U}{2}\right)\right],
$$

where we can see that $W_{i, i+1}$ depends not only on $\Delta V$ in the usual way, but also on the average $\left(V_{i+1}+V_{i}\right) / 2$ through parameter $\theta$. In the next paragraphs we show that the equilibrium solution does not depend on $\theta$; this parameter has influence only in the out of equilibrium behavior.

From the transition probabilities we can obtain the following expression for the current in the continuous limit [27]:

$$
J=-D_{0} e^{-\beta \theta V}\left(c \beta \frac{\partial U}{\partial x}+c \beta \frac{\partial V}{\partial x}+\frac{\partial c}{\partial x}\right)
$$

Both the potential $V$ and the activity coefficient $\gamma$ contain the same information, since both represent the interactions. In the absence of interactions we assume that $V=0$ and $\gamma=1$.

\section{A. Relation between $V$ and $\gamma$}

Eq. (1) gives the chemical potential as a function of concentration. From this equation we can immediately write the concentration as a function of temperature and chemical potential:

$$
c=c_{0} \exp \left[-\beta\left(k_{B} T \ln \gamma+\tilde{\mu}^{\mathrm{ref}}-\tilde{\mu}\right)\right],
$$

where $\tilde{\mu}=\mu / N_{A}$ is the chemical potential per particle (and $R=k_{B} N_{A}$ ). This expression is not explicit, since $\gamma$ depends on $c$ (let us note that, according to the definition of the chemical potential (1), the reference values 
$c_{0}$ and $\tilde{\mu}^{\text {ref }}$ do not depend on the concentration $c$ ). Let us compare it with the equilibrium concentration that is obtained from taking $J=0$ in (5):

$$
c=c_{0}^{\prime} e^{-\beta(U+V-\tilde{\mu})}
$$

where $c_{0}^{\prime}$ is another reference concentration that, in this case, is originated in an integration constant. It can be immediately verified that (7) is an equilibrium solution by replacing it in (5), and we can also see that it is independent of $\theta$. From (6) and (7) we have

$$
U+V-k_{B} T \ln c_{0}^{\prime} / c_{0}=k_{B} T \ln \gamma+\tilde{\mu}^{\mathrm{ref}} .
$$

Let us consider the case in which the concentration is small enough so that $V=0$ and $\gamma=1$, then we have

$$
U-k_{B} T \ln c_{0}^{\prime} / c_{0}=\tilde{\mu}^{\mathrm{ref}}
$$

a relationship that is independent of $c$, and that can be put back into the original expression (8). By doing this, we obtain the relationship between the mean field potential and the activity coefficient:

$$
V=k_{B} T \ln \gamma
$$

This equation was derived for a system in equilibrium, with fixed values of temperature and chemical potential. We are however interested in out of equilibrium diffusion processes. We can use Eq. (10) in the expression (5) for the current, even for $J \neq 0$, as long as the local thermal equilibrium approximation holds. This approximation holds as long as we have smooth variations between neighboring cells (each cell can be taken as a system in equilibrium, even though the whole system is out of equilibrium); as mentioned before, the cell length should be much smaller than the spatial variations of the concentration. This is a standard approximation in classical irreversible thermodynamics.

\section{B. Collective diffusion coefficient $D$}

Using the relation between $V$ and $\gamma(10)$ in the expression for the current (5), we have

$$
J=-\underbrace{D_{0} \gamma^{-\theta} c \beta \frac{\partial U}{\partial x}}_{J_{\mathrm{drift}}}-\underbrace{D_{0} \gamma^{-\theta}\left(1+\frac{\partial \ln \gamma}{\partial \ln c}\right) \frac{\partial c}{\partial x}}_{J_{\mathrm{diff}}}
$$

where we can recognize the drift current $J_{\text {drift }}$ and the



$$
D=D_{0} \gamma^{-\theta}\left(1+\frac{\partial \ln \gamma}{\partial \ln c}\right)
$$

expression that has the shape of the Darken equation (2). In order to complete the connection with (2), it is necessary to calculate the single particle diffusion coefficient.

\section{Single particle diffusion coefficient $D^{*}$}

The mean square displacement of a tagged particle is

$$
\left\langle(\Delta x)^{2}\right\rangle=2 D^{*} t
$$

We can calculate $\left\langle(\Delta x)^{2}\right\rangle$ using the transition probabilities (3) if we consider a time interval $\Delta t$ small enough so that jumps occur only between neighboring cells. Let us suppose that the tagged particle is in cell $i$ at time 0 . The probabilities to have a jump to the right or left after a time $\Delta t$ are $W_{i, i+1} \Delta t$ and $W_{i, i-1} \Delta t$ respectively. With this information we can calculate $\left\langle(\Delta x)^{2}\right\rangle$ after a time $\Delta t$ :

$$
\begin{aligned}
\left\langle(\Delta x)^{2}\right\rangle= & a^{2}\left(W_{i, i+1}+W_{i, i-1}\right) \Delta t \\
= & a^{2} P e^{-\beta\left[(\theta-1) V_{i}-U_{i}\right] / 2}\left(e^{-\beta\left[(\theta+1) V_{i+1}+U_{i+1}\right] / 2}\right. \\
& \left.+e^{-\beta\left[(\theta+1) V_{i-1}+U_{i-1}\right] / 2}\right) \Delta t
\end{aligned}
$$

Now we suppose that the quantities that describe the system have smooth spatial variations and proceed to find the continuous limit: we replace $V_{i} \rightarrow V, V_{i+1} \rightarrow$ $V+a \frac{\partial V}{\partial x}, V_{i-1} \rightarrow V-a \frac{\partial V}{\partial x}$ and similar replacements for the external potential $U$. We obtain

$$
\begin{aligned}
\left\langle(\Delta x)^{2}\right\rangle= & a^{2} P e^{-\beta[(\theta-1) V-U] / 2} e^{-\beta[(\theta+1) V+U] / 2} \\
& \times\left\{\exp \left[-\beta a\left((\theta+1) \frac{\partial V}{\partial x}+\frac{\partial U}{\partial x}\right) / 2\right]\right. \\
& \left.+\exp \left[\beta a\left((\theta+1) \frac{\partial V}{\partial x}+\frac{\partial U}{\partial x}\right) / 2\right]\right\} \Delta t \\
= & a^{2} P e^{-\beta \theta V}\left[2+\mathcal{O}\left(a^{2}\right)\right] \Delta t \\
= & a^{2} P \gamma^{-\theta} 2 \Delta t
\end{aligned}
$$

where, in the last step, terms of higher order in $a$ were neglected and Eq. (10) was used. This result implies that

$$
D^{*}=D_{0} \gamma^{-\theta}
$$

Using this expression for the single particle diffusion coefficient in (12), we recover the Darken equation (2). The derivation of the Darken equation starting from the transition probabilities proposed in the mean field treatment [27] is a result that supports this procedure.

In the small concentration limit, both $D$ and $D^{*}$ take the value $D_{0}=P a^{2}$, where $D_{0}$, as mentioned before, is the free diffusion coefficient.

\section{NUMERICAL VALIDATION}

A standard criterion that guarantees the validity of a mean field theory is given by the condition $\left\langle\Delta n_{i}^{2}\right\rangle /\left\langle n_{i}\right\rangle^{2} \ll 1$, i.e., the relative fluctuations of the order parameter should be much smaller than 1 ; this is known as the Ginzburg criterion [32, p. 460].

Now let us consider the case of boson statistics. The Bose-Einstein distribution can be obtained in this classical context using an appropriate statistical potential [32, 
p. 138]. Considering that the fluctuations of the classical distribution are "normal", we have that boson's fluctuations are above normal [32, p. 151]: $\left\langle\Delta n_{i}^{2}\right\rangle /\left\langle n_{i}\right\rangle^{2}=$ $1 /\left\langle n_{i}\right\rangle+1$. This means that the Ginzburg criterion is never fulfilled for bosons. Nevertheless, we show below, through numerical simulations, that the mean field approach gives correct results for boson statistics. The reason is that the Ginzburg criterion was proposed for systems with a phase transition, where there is a relationship between large fluctuations and large correlation length. In our case, there is no phase transition; there are large fluctuations, but there is no fluctuation correlation between neighboring cells. Therefore, we propose below a different validity criterion based on the specific approximations that are performed in the present approach.

Let us call $\Phi\left(n_{i}\right)$ the effective attractive potential (or statistical potential) for $n_{i}$ particles in cell $i$ that produces boson statistics. It can be shown (see Ref. [27]) that it takes the form

$$
\Phi\left(n_{i}\right)=-\beta^{-1} \ln n_{i} !
$$

The mean field potential $V$, for one particle in cell $i$, is

$$
V\left(n_{i}\right)=-\beta^{-1} \ln \left(1+n_{i}\right) .
$$

This expression can be derived from $\Phi$; see Eq. (38) in Ref. [27], where the mean field potential is written as a function of the average number of particles $n=\left\langle n_{i}\right\rangle$.

Numerical simulations of a system of bosons, including an external force, can be performed using (17) or (18). In the first case, transition probabilities are written using, for example, the Metropolis algorithm. In the second case, transition probabilities are given by (3), and they depend on parameter $\theta$. We have considered $\theta=-1,0$ and 1 .

Numerical results of the equilibrium concentration using (17), with the Metropolis algorithm, show no systematic error when compared with the Bose-Einstein distribution; as shown in Fig. 1 (plus sign symbols).

On the other hand, numerical results using the transition probabilities of the mean field theory (3), also show no systematic error for $\theta=1$, and small deviations from the theoretical result for other values of $\theta$; see Fig. 1 . The results are correct despite the large value of the fluctuations and the violation of the Ginzburg criterion.

Nevertheless, the fluctuations influence the deviations observed for $\theta=-1$ and 0 . In order to elucidate the origin of these deviations, we have to analyze the approximations applied in the method.

\section{A. Decorrelation error}

The starting point in the derivation of the current (5) is the following expression:

$$
J_{i, i+1}=n_{i} W_{i, i+1}-n_{i+1} W_{i+1, i}
$$

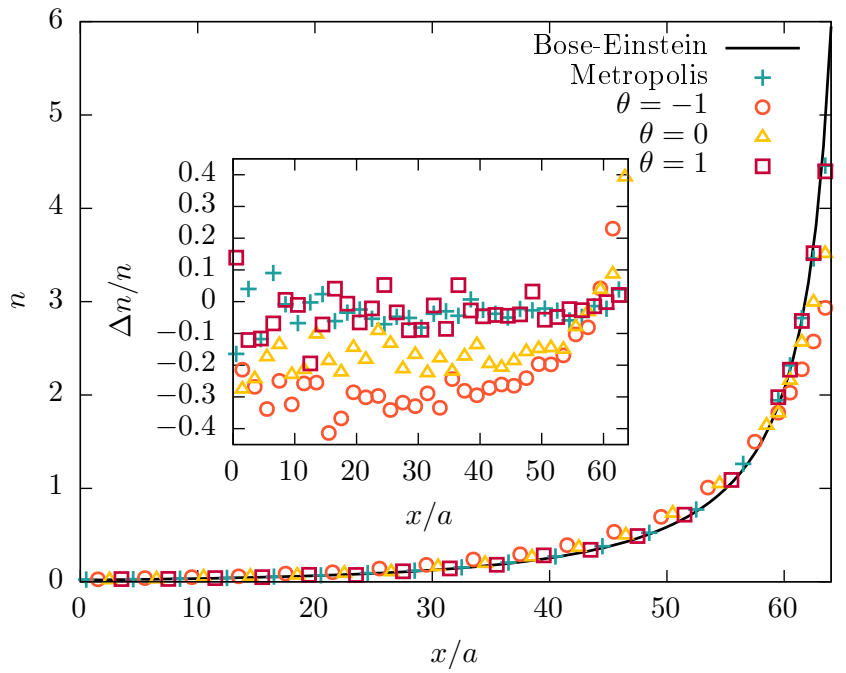

FIG. 1. Equilibrium particle concentration $n$ against position $x / a$. A positive force, with $\beta F a=0.06$, is applied. The curve corresponds to the Bose-Einstein distribution. Numerical results were obtained with transition probabilities given by (3) with $\theta=-1$ (circles), $\theta=0$ (triangles) and $\theta=1$ (squares). Numerical results using the Metropolis algorithm, with the potential (17), are also shown (plus signs). Other parameters: number of particles, $N=32$; system size, $L=64 a$; number of samples, 100; and Monte Carlo steps, $10^{7}$. Inset: Relative error $\Delta n / n$ (difference between the numerical results for $n$ and the expected Bose-Einstein distribution) as a function of the position.

where $J_{i, i+1}$ is the current between cells $i$ and $i+1$ for given values of the occupation numbers $n_{i}$ and $n_{i+1}$, and the corresponding transition probabilities. The current $J$ is obtained by applying a sample average:

$$
\begin{aligned}
J & =\left\langle J_{i, i+1}\right\rangle=\left\langle n_{i} W_{i, i+1}\right\rangle-\left\langle n_{i+1} W_{i+1, i}\right\rangle \\
& \simeq\left\langle n_{i}\right\rangle \bar{W}_{i, i+1}-\left\langle n_{i+1}\right\rangle \bar{W}_{i+1, i}
\end{aligned}
$$

where we use $\bar{W}_{i, i+1}=W_{i, i+1}\left(\left\langle n_{i}\right\rangle,\left\langle n_{i+1}\right\rangle\right)$, and a similar expression for $\bar{W}_{i+1, i}$, in order to reduce the notation. The main approximation of the procedure was performed in the last step of the previous equation, where fluctuations were neglected. We wish to know the error magnitude introduced by this approximation in the presence of the large fluctuations in boson occupation number. Since we analyze equilibrium situations, for which $J=0$, it is convenient to evaluate the relative error of only one of the terms in the right hand side of Eq. (20). We define the error introduced by decorrelation as

$$
\eta=\frac{\left\langle n_{i} W_{i, i+1}\right\rangle-\left\langle n_{i}\right\rangle \bar{W}_{i, i+1}}{\left\langle n_{i}\right\rangle \bar{W}_{i, i+1}}
$$

We can evaluate the decorrelation error $\eta$ both numerically and analytically. For example, for $\theta=-1$, using Eqs. (3) and (18), we have $W_{i, i+1}=P e^{-\beta \Delta U / 2}\left(1+n_{i}\right)^{-1}$; 


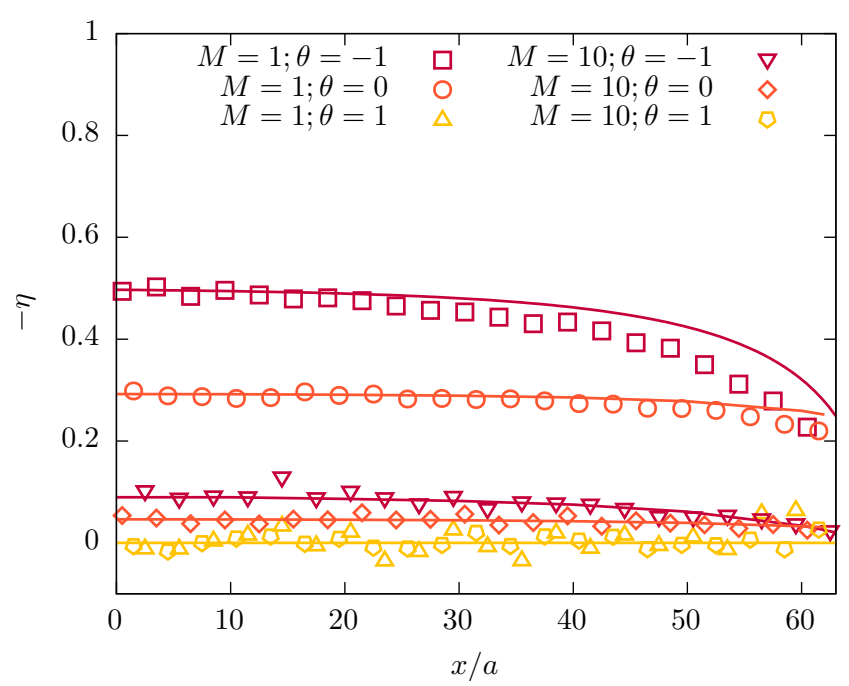

FIG. 2. Decorrelation error $\eta$ against position $x / a$ in equilibrium for different values of $\theta$ and $M$. For $M=1$ : $\theta=-1$ (squares), $\theta=0$ (circles) and $\theta=1$ (up triangles); for $M=10: \theta=-1$ (down triangles), $\theta=0$ (rhomboids) and $\theta=1$ (pentagons). Curves correspond to the evaluation of Eqs. (23), (24) and (25) for $M=1$; for $M=10$, the curves are evaluated using the probability distribution (26).

and the corresponding decorrelation error is

$$
\eta_{\theta=-1}=\frac{\left\langle\frac{n_{i}}{1+n_{i}}\right\rangle-\frac{\left\langle n_{i}\right\rangle}{1+\left\langle n_{i}\right\rangle}}{\frac{\left\langle n_{i}\right\rangle}{1+\left\langle n_{i}\right\rangle}} .
$$

Averages can be computed using the probability distribution for bosons that, in terms of the average $\left\langle n_{i}\right\rangle$, is given by $P\left(n_{i}\right)=\left\langle n_{i}\right\rangle^{n_{i}} /\left(1+\left\langle n_{i}\right\rangle\right)^{n_{i}+1}$; see, e.g., [32, p. 152]. The solution in this case is

$$
\eta_{\theta=-1}=\frac{1+\left\langle n_{i}\right\rangle}{\left\langle n_{i}\right\rangle^{2}}\left(\ln \left(\frac{1}{1+\left\langle n_{i}\right\rangle}\right)+\left\langle n_{i}\right\rangle\right)-1 .
$$

Similarly, for $\theta=0$ we have

$$
\eta_{\theta=0}=\frac{\left\langle n_{i} \sqrt{\frac{1+n_{i+1}}{1+n_{i}}}\right\rangle-\left\langle n_{i}\right\rangle \sqrt{\frac{1+\left\langle n_{i+1}\right\rangle}{1+\left\langle n_{i}\right\rangle}}}{\left\langle n_{i}\right\rangle \sqrt{\frac{1+\left\langle n_{i+1}\right\rangle}{1+\left\langle n_{i}\right\rangle}}} .
$$

And, for $\theta=1$,

$$
\eta_{\theta=1}=\frac{\left\langle n_{i}\left(1+n_{i+1}\right)\right\rangle-\left\langle n_{i}\right\rangle\left(1+\left\langle n_{i+1}\right\rangle\right)}{\left\langle n_{i}\right\rangle\left(1+\left\langle n_{i+1}\right\rangle\right)}=0,
$$

equal to 0 because fluctuations in $i$ and in $i+1$ are uncorrelated: $\left\langle n_{i} n_{i+1}\right\rangle=\left\langle n_{i}\right\rangle\left\langle n_{i+1}\right\rangle$. This result explains why we found no systematic error in the equilibrium concentration against position for $\theta=1$; see Fig. 1 .

Numerical and analytical results of the decorrelation error for the three values of $\theta(-1,0$ and 1$)$ can be seen in Fig. 2.

\section{B. Modified system to reduce fluctuations}

The systematic error that appears for $\theta=-1$ and 0 can be reduced considering a modified system [27]. This new system preserves the boson statistics, but a larger number of particles is introduced in order to reduce fluctuations. Let us call $m_{i}$ the new occupation number. It is related to $n_{i}$ by $m_{i}=M n_{i}$, where $M$ is an integer. It can be shown that, in this case, the partition function is $\mathcal{Z}_{M}=\left(1+\left\langle m_{i}\right\rangle / M\right)^{M}$ and the probability distribution for $m_{i}$ is (see the appendix for more details)

$$
P\left(m_{i}\right)=\left(\begin{array}{c}
m_{i}+M-1 \\
m_{i}
\end{array}\right) M^{M} \frac{\left\langle m_{i}\right\rangle^{m_{i}}}{\left(M+\left\langle m_{i}\right\rangle\right)^{m_{i}+M}} .
$$

Let us consider the decorrelation error when it takes its larger absolute value, for $\theta=-1$. Now it takes the form

$$
\eta_{\theta=-1}=\frac{\left\langle\frac{m_{i}}{M+m_{i}}\right\rangle-\frac{\left\langle m_{i}\right\rangle}{M+\left\langle m_{i}\right\rangle}}{\frac{\left\langle m_{i}\right\rangle}{M+\left\langle m_{i}\right\rangle}} .
$$

One can expect that $\eta_{\theta=-1} \rightarrow 0$ when $M \rightarrow \infty$, since the relevant values of $m_{i}$, or $\left\langle m_{i}\right\rangle$, are negligible with respect to $M$. In Fig. 2, we show numerically and analytically that this indeed happens as the decorrelation error becomes smaller as $M$ is increased, thus more accurate results of the equilibrium concentration are obtained for any value of $\theta$.

\section{CONCLUSIONS}

We checked the validity of the mean field approach for diffusion with interaction introduced in Ref. [27] from two different perspectives.

In Sect. II we showed that this treatment can be used to reproduce the Darken equation that relates the collective diffusion coefficient $D$ to the single particle diffusion coefficient $D^{*}$. The relationship depends on the interactions, through the activity coefficient, and on the concentration. The Darken equation was originally derived from the linear relationship between particle current and its associated thermodynamic force, the chemical potential gradient. Here, instead, the equation was obtained from the transition probabilities $W_{i, i+i}$ (derived in [27]) that, in turn, depend on the mean field potential for one particle produced by the presence of the other particles. The context in which the equation is obtained is also different. Darken assumed a mixture of two atomic species with the same atomic volume. Here, no assumption is made on the volume of the particles, but it is assumed that the description can be reduced to the diffusion of only one species in a stationary environment.

In Sect. III we performed a numerical verification of the mean field approach using the interactions that reproduce the boson statistics. This is a particularly appropriate case to test the method since bosons have above normal fluctuations. The Ginzburg criterion, usually considered for the validity of a mean field theory, is not fulfilled 
due to the presence of large fluctuations. Nevertheless, the criterion is applied to systems with a phase transition, where large fluctuations are associated to a large correlation length. In our case there is no phase transition, and there is no correlation between neighboring cells. The numerical results of the equilibrium distribution are correct despite the presence of large fluctuations. We noted a systematic error when the interpolation parameter $\theta$ is equal to -1 and 0 , and no systematic error for $\theta=1$. This difference was explained through the decorrelation error $\eta$, defined in Eq. (21); this quantity is a measure of the error introduced in the current when fluctuations are neglected. We also showed that this error can be arbitrarily reduced in an alternative system that also reproduces boson statistics. In this system, the number of particles is increased by a factor $M$, and fluctuations are reduced.

\section{APPENDIX}

In this appendix we describe in more detail the derivation of the partition function and the probability distribution for the system with $m_{i}=M n_{i}$ particles introduced in Sect. IIIB. For the original system with $n_{i}$ particles, the partition function of bosons is

$$
\mathcal{Z}_{1}=\sum_{n_{i}=0}^{\infty} e^{-\delta n_{i}}=\frac{1}{1-e^{-\delta}}
$$

where $\delta=\beta(U-\mu)$, with $U$ being an external potential. Since $m_{i}=M n_{i}$, we have that $\left\langle m_{i}\right\rangle=M\left\langle n_{i}\right\rangle$ and

$$
\left\langle m_{i}\right\rangle=-M \frac{\partial \ln \mathcal{Z}_{1}}{\partial \delta}=-\frac{\partial \ln \mathcal{Z}_{1}^{M}}{\partial \delta} .
$$

The new partition function for $m_{i}$ is

$$
\begin{aligned}
\mathcal{Z}_{M} & =\mathcal{Z}_{1}^{M}=\left(1-e^{-\delta}\right)^{-M} \\
& =\sum_{m_{i}=0}^{\infty}\left(\begin{array}{c}
M+m_{i}-1 \\
m_{i}
\end{array}\right) e^{-\delta m_{i}}
\end{aligned}
$$

where the binomial series was used. We have that each term in the sum of (30) is proportional to the probability of having $m_{i}$ particles. Then, the probability distribution is

$$
P\left(m_{i}\right)=\frac{1}{\mathcal{Z}_{M}}\left(\begin{array}{c}
M+m_{i}-1 \\
m_{i}
\end{array}\right) e^{-\delta m_{i}}
$$

Knowing that $e^{-\delta}=\left\langle n_{i}\right\rangle /\left(1+\left\langle n_{i}\right\rangle\right)=\left\langle m_{i}\right\rangle /\left(M+\left\langle m_{i}\right\rangle\right)$ we obtain

$$
\mathcal{Z}_{M}=\left(1+\left\langle m_{i}\right\rangle / M\right)^{M}
$$

and Eq. (26) for the probability distribution.

\section{ACKNOWLEDGMENTS}

The authors acknowledge H. Mártin, N. A. Rodríguez and A. Grela for illuminating discussions regarding the effects of interaction on diffusion. This work was partially supported by Consejo Nacional de Investigaciones Científicas y Técnicas (CONICET, Argentina, PIP 112 $20150100021 \mathrm{CO})$.
[1] L. S. Darken, Diffusion, mobility and their interrelation through free energy in binary metallic systems, Trans. AIME 175, 184 (1948).

[2] S. Sridhar, A Commentary on "Diffusion, Mobility and Their Interrelation through Free Energy in Binary Metallic Systems," L.S. Darken: Trans. AIME, 1948, vol. 175, p. 184ff, Metallurgical and Materials Transactions A 41, 543 (2010).

[3] H. De Voe, Thermodynamics and Chemistry, Pearson Education, 2001.

[4] P. Atkins and J. de Paula, Physical Chemistry, Oxford University Press, 2006.

[5] R. Ferrando, E. Scalas and M. Torri, Projection-operator route to the generalized Darken equation, Phys. Lett. A 186, 415 (1994).

[6] G. K. Batchelor, Brownian diffusion of particles with hydrodynamic interaction, J. Fluid Mech. 74, 1-29 (1976).

[7] T. J. Murphy and J. L. Aguirre, Brownian Motion of N Interacting Particles. I. Extension of the Einstein Diffusion Relation to the N-Particle Case, J. Chem. Phys. 57, 2098 (1972).
[8] B. U. Felderhof, Diffusion of interacting Brownian particles, J. Phys. A: Math. Gen. 11, 929 (1978).

[9] R. Finsy, A. Devriese and H. Lekkerkerker, Light Scattering Study of the Diffusion of Interacting Particles, J. C. S. Faraday II 76, 767-775 (1980).

[10] T. Ohtsuki and K. Okano, Diffusion coefficients of interacting Brownian particles, J. Chem. Phys. 77, 1443 (1982).

[11] H. N. W. Lekkerkerker and J. K. G. Dhont, On the calculation of the self-diffusion coefficient of interacting Brownian particles, J. Chem. Phys. 80, 5790 (1984).

[12] B. Cichocki and B. U. Felderhof, Self-diffusion in suspensions of interacting Brownian particles, Phys. Rev. A 42, 6024 (1990).

[13] S. E. Guidoni and C. M. Aldao, On diffusion, drift and the Einstein relation, Eur. J. Phys. 23, 395-402 (2002).

[14] G. L. Aranovich and M. D. Donohue, Diffusion Equation for Interacting Particles, J. Phys. Chem. B 109, 16062 (2005).

[15] S. Savel'ev, F. Marchesoni, A. Taloni and F. Nori, Diffusion of interacting Brownian particles: Jamming and 
anomalous diffusion, Phys. Rev. E 74, 021119 (2006).

[16] M. J. Simpson, K. A. Landman and B. D. Hughes, Multispecies simple exclusion processes, Physica A 388, 399 (2009).

[17] M. Bruna and S. J. Chapman, Excluded-volume effects in the diffusion of hard spheres, Phys. Rev. E 85, 011103 (2012).

[18] Chikashi Arita, P. L. Krapivsky and Kirone Mallick, Generalized exclusion processes: Transport coefficients, Phys. Rev. E 90, 052108 (2014).

[19] A. Pototsky, A. J. Archer, M. Bestehorn, D. Merkt, S. Savel'ev, and F. Marchesoni, Collective shuttling of attracting particles in asymmetric narrow channels, Phys. Rev. E 82, 030401(R) (2010).

[20] A. Pototsky, A. J. Archer, S. E. Savel'ev, U. Thiele, and F. Marchesoni, Ratcheting of driven attracting colloidal particles: Temporal density oscillations and current multiplicity, Phys. Rev. E 83, 061401 (2011).

[21] D. A. Reed and G. Ehrlich, Surface diffusion, atomic jump rates and thermodynamics, Surface Science 102, 588 (1981).

[22] R. Gomer, Diffusion of adsorbates on metal surfaces, Rep. Prog. Phys. 53, 917 (1990).

[23] A. Pimpinelli and J. Villain, Physics of Crystal Growth, Cambridge University Press, Cambridge, 1998.
[24] M.C. Tringides and z. Chvoj (eds.), Collective Diffusion on Surfaces: Correlation Effects and Adatom Interactions, Springer Science, Prague, 2000.

[25] T. Ala-Nissila, R. Ferrando and S. C. Ying, Collective and single particle diffusion on surfaces, Advances in Physics, 51, 949-1078 (2002)

[26] K. Wu, X. Li, C. Wang, W. Yu and Z. Chen, Model for surface diffusion of adsorbed gas in nanopores of shale gas reservoirs, Ind. Eng. Chem. Res. 54, 3225 (2015).

[27] G. Suárez, M. Hoyuelos and H. Mártin, Mean-field approach for diffusion of interacting particles, Phys. Rev. E 92, 062118 (2015)

[28] S. Savel'ev, F. Marchesoni, and F. Nori, Controlling Transport in Mixtures of Interacting Particles using Brownian Motors, Phys. Rev. Lett. 91, 010601 (2003).

[29] S. Savel'ev, F. Marchesoni, and F. Nori, Manipulating Small Particles in Mixtures far from Equilibrium, Phys. Rev. Lett. 92, 160602 (2004).

[30] S. Savel'ev, F. Marchesoni, and F. Nori, Stochastic transport of interacting particles in periodically driven ratchets, Phys. Rev. E 70, 061107 (2004).

[31] W. Janke, Monte Carlo Methods in Classical Statistical Physics, in Computational Many-Particle Physics (H, Fehske, R. Schneider and A. Weiße, eds.), Springer, 2007.

[32] R. K. Pathria and P. D. Beale, Statistical Mechanics (3rd edition), Elsevier Butterworth-Heinemann, Oxford, 2011. 